\title{
Quantum Hall effects of graphene with multiorbitals: Topological numbers, Boltzmann conductance, and semiclassical quantization
}

\author{
Masao Arai* \\ Computational Materials Science Center, National Institute for Materials Science, Tsukuba, Ibaraki 305-0044, Japan
}

\author{
Yasuhiro Hatsugai ${ }^{\dagger}$ \\ Institute of Physics, University of Tsukuba, Tsukuba, Ibaraki 305-8571, Japan
}

(Received 5 December 2008; published 18 February 2009)

\begin{abstract}
Hall conductance $\sigma_{x y}$ as the Chern numbers of the Berry connection in the magnetic Brillouin zone is calculated for a realistic multiband tight-binding model of graphene with a nonorthogonal basis. It is confirmed that the envelope of $\sigma_{x y}$ coincides with a semiclassical result when the magnetic field is sufficiently small. The Hall resistivity $\rho_{x y}$ from the weak-field Boltzmann theory also explains the overall behavior of the $\sigma_{x y}$ if the Fermi surface is composed of a single energy band. The plateaux of $\sigma_{x y}$ are explained from semiclassical quantization and necessary modification is proposed for the Dirac fermion regimes.
\end{abstract}

DOI: 10.1103/PhysRevB.79.075429

PACS number(s): 73.43.-f, 03.65.Sq, 73.21.-b

\section{INTRODUCTION}

The quantum Hall effect (QHE) has been one of the important subjects in condensed-matter physics for several decades. Wide ranges of experimental and theoretical studies have been devoted to this subject. In the early stage of these efforts, the topological aspects of quantization have been firmly established. ${ }^{1-7}$ The topological formulation of quantized Hall conductance ${ }^{8}$ has been utilized numerically to explore the exotic nature of electrons on two-dimensional lattice models.

Recently, electrons on a honeycomb lattice have attracted theoretical attention because energy bands on this lattice are described by massless Dirac dispersion near the center of the bands. The dispersion results in anomalous QHE $\sigma_{x y}$ $=(2 N+1) e^{2} / h(N$ : integer $)$ per spin., ${ }^{9} 10$ Such anomalous QHE was observed experimentally in graphene, which is monolayer graphite. ${ }^{11,12}$ The topological aspect of QHE in graphene has been studied by using a single-orbital tightbinding model on a honeycomb lattice. ${ }^{13,14}$ It has been shown that anomalous QHE persists up to the van Hove singularities. ${ }^{14}$ At the singularities, $\sigma_{x y}$ jumps discontinuously and normal QHE $\sigma_{x y}=N e^{2} / h$ is recovered around band-edge regions. These results suggest that band structure affects QHE both qualitatively and quantitatively. Thus, it is an interesting question to investigate the relation between band structure and $\sigma_{x y}$.

In this paper, we study relations between band structure and quantized $\sigma_{x y}$ by using a realistic tight-binding model of graphene as an example. Up to now, numerical calculations of quantized $\sigma_{x y}$ have been rather limited to simple tightbinding models and realistic band structure was beyond the scope of these studies. Another purpose of this study is to demonstrate that the topological formulation of $\sigma_{x y}$ (Refs. 1 and 8) can be applicable to realistic band structures.

\section{MODEL AND CALCULATION METHODS}

We use a tight-binding model for graphene with $s$ and $p$ orbitals on carbon atoms. ${ }^{15}$ The spin degeneracy is ignored for simplicity. Each orbital $|i \xi\rangle$ is labeled by a site index $i$ and an orbital index $\xi$. Transfer $t_{i \xi, j \xi^{\prime}}$ and overlap integrals $s_{i \xi, j \xi^{\prime}}$ between nearest-neighbor atoms are considered by a Slater-Koster approximation. The onsite energy $\varepsilon_{i \xi}, t_{i \xi, j \xi^{\prime}}$, and $s_{i \xi, j \xi^{\prime}}$ are obtained from the parameters in Refs. 15 and 16. With eight orbitals in a unit cell, energy-band dispersion is calculated as shown in Fig. 1. In actual graphene, electrons are occupied up to $\varepsilon=0$. Around this energy, band dispersions are well approximated by the massless Dirac cones, which are the origin of several interesting physical properties including anomalous quantization of Hall conductance. The massless Dirac dispersions are also realized around $\varepsilon$ $\sim-14 \mathrm{eV}$. In addition, several van Hove singularities are clearly seen in the total density of states (DOS).

The uniform magnetic field is introduced as

$$
\left\langle i \xi|\hat{H}| j \xi^{\prime}\right\rangle=e^{i \theta_{i j}} t_{i \xi, j \xi^{\prime}},\left\langle i \xi \mid j \xi^{\prime}\right\rangle=e^{i \theta_{i j}} s_{i \xi, j \xi^{\prime}}
$$

so that they satisfy $\Sigma_{\text {closed loop }} \theta_{i j}=2 \pi$ (flux quanta in the loop). Under the uniform magnetic field, original translational symmetry is broken. The system is then characterized by the magnetic flux $\phi=B \Omega / \varphi_{0}$, where $\Omega$ is the area of a unit cell and $\varphi_{0}=h c / e$ is the quantized magnetic flux. When $\phi$ is a rational number $p / q$, we can define a magnetic unit cell whose area is $q$ times larger than that of the original cell. Then, the extended Bloch theorem holds for the magnetic unit cell and eigenstates are labeled by a wave number $\boldsymbol{k}$. Thus, an energy band splits into $q$ subbands by the uniform field. If the chemical potential is located within an energy gap, quantized Hall conductance $\sigma_{x y}$ is given as

$$
\sigma_{x y}=-\frac{e^{2}}{h} c_{F}(\mu),
$$

where $c_{F}$ is a topological integer called the Chern number defined for the filled bands. It is calculated by discretizing the Brillouin zone into mesh $\left\{\boldsymbol{k}_{\ell}\right\}$ as $1,3,8$

$$
c_{F}(\mu)=\frac{1}{2 \pi} \sum_{\ell} F\left(\boldsymbol{k}_{\ell}\right),
$$




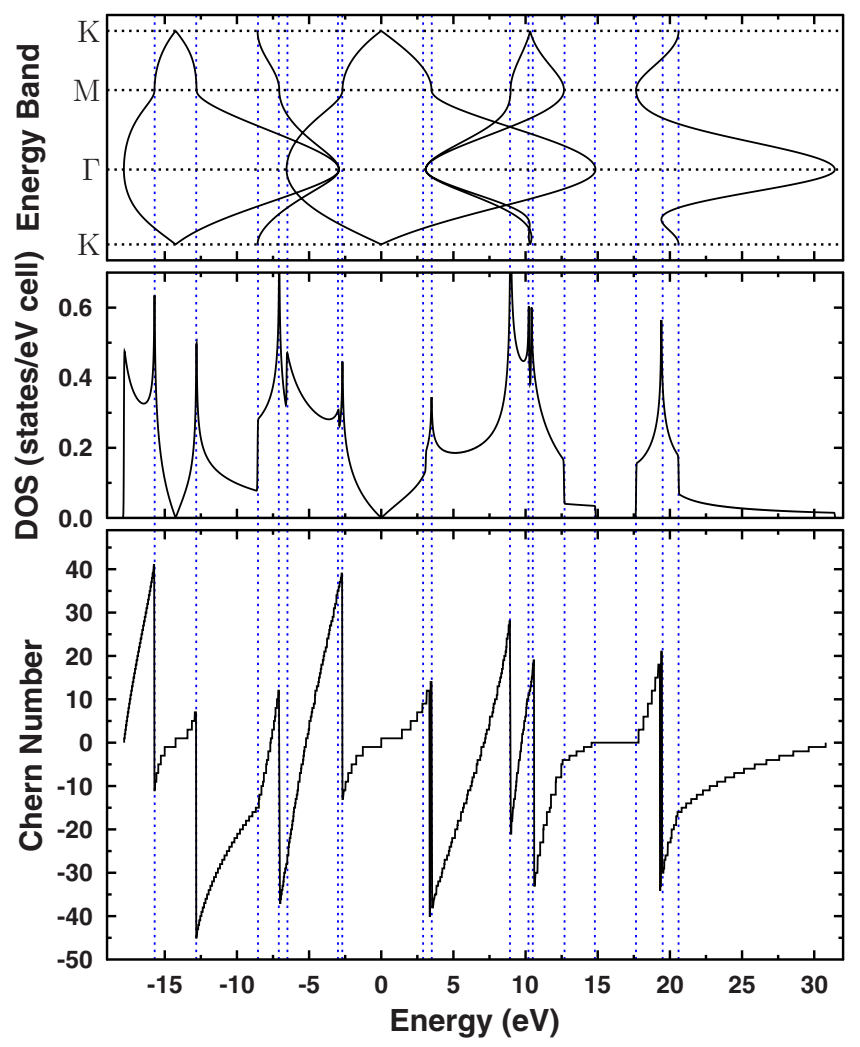

FIG. 1. (Color online) The lower panel shows the calculated Chern number $\left(\sigma_{x y}\right)$ for $\phi=1 / 53$ as a function of the chemical potential. The upper and middle panels present energy bands and total density of states when magnetic field is absent. Vertical dashed lines indicate positions of van Hove singularities.

$$
\begin{gathered}
F(\boldsymbol{k})=\operatorname{Arg} u_{x}(\boldsymbol{k}) u_{y}\left(\boldsymbol{k}+\Delta \boldsymbol{k}_{x}\right)\left[u_{x}\left(\boldsymbol{k}+\Delta \boldsymbol{k}_{y}\right) u_{y}(\boldsymbol{k})\right]^{*}, \\
u_{\mu}(\boldsymbol{k})=\operatorname{det}[\Psi(\boldsymbol{k})]^{\dagger} \Psi\left(\boldsymbol{k}+\Delta \boldsymbol{k}_{\mu}\right), \\
\Psi(\boldsymbol{k})=\left[\left|\psi_{1}(\boldsymbol{k})\right\rangle, \cdots,\left|\psi_{M}(\boldsymbol{k})\right\rangle\right],
\end{gathered}
$$

where $\Delta \boldsymbol{k}_{\nu}$ is a discretized momentum along the $\nu$ direction, $\left|\psi_{m}(\boldsymbol{k})\right\rangle$ is a Bloch state of the band index $m$, and $m=M$ is the highest occupied energy band below the chemical potential $\mu$. (See Ref. 8 for details.)

This formulation was successfully applied to the quantum Hall effect on a disorder system, ${ }^{17}$ graphene, ${ }^{14}$ and a chiral two-dimensional system. ${ }^{18}$ When the field $\phi=p / q$ is sufficiently small, $p$ subbands are grouped together in general. These grouped bands correspond to a Landau band of nearly free electrons. Hereafter, we treat the field $\phi=1 / q$, which enables us to consider the weak-field limit easily.

\section{RESULTS AND DISCUSSIONS}

In Fig. 1, we show the calculated Chern number $c_{F}$ with $\phi=1 / 53$ as a function of the chemical potential. The $c_{F}$ of adjacent gaps are connected by a straight line as guide for the eyes. In some energy regions, several Landau bands are grouped together with very narrow energy gaps, which are typically smaller than $10^{-1} \mathrm{meV}$ for $\phi=1 / 53$ and rapidly

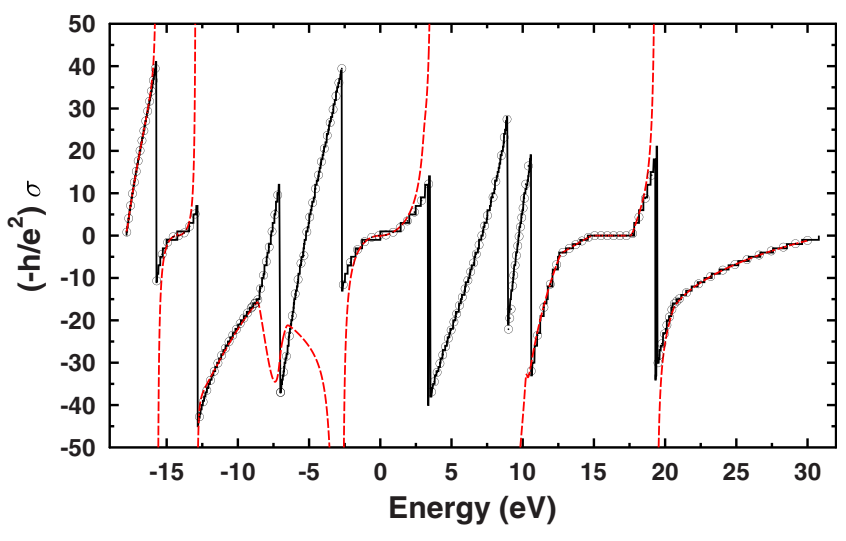

FIG. 2. (Color online) Comparison of the Hall conductance obtained from Chern number with semiclassical formulations. The solid line shows the $\sigma_{x y}$ obtained from Chern number and open circles indicate that from semiclassical theory with clean limit. The dashed line is $-\rho_{x y}^{-1}$ obtained from weak-field limit of Boltzmann theory.

diminish as $\phi$ decreases. In such a case, we found that Chern numbers corresponding to these small gaps are sensitive to the choice of $\phi$. Thus, they are not physically relevant and we omit them from Fig. 1. The plateaux are most clearly seen at Dirac fermion regions because of large energy gaps between Landau bands. The $c_{F}$ increases by 1,2 , or 3 between adjacent (well-developed) energy gaps. If these steps are smeared, coarse grained $\sigma_{x y}$ behaves as a continuous function except for several energies where the $c_{F}$ changes with large numbers.

We found that the large discontinuities in $c_{F}$ can be attributed to topological changes in the Fermi surface when the magnetic field is absent. For example, the discontinuity at $\varepsilon \approx-15.7 \mathrm{eV}$ is caused by a transformation of the Fermi surface from an electron pocket around $\Gamma$ points to two hole pockets around $K$ points [see Figs. 3(a) and 3(b)]. Such topological changes are also recognized as a van Hove singularity in the DOS. Other discontinuities also correspond to similar topological changes in the Fermi surface.

We compare the global behavior of $\sigma_{x y}$ with a semiclassical theory. When Fermi surface consists of closed curves, the Hall conductance $\sigma_{x y}$ for the clean limit can be calculated as ${ }^{19,20}$

$$
\sigma_{x y}^{\mathrm{sc}}(\mu)=-\frac{e c}{(2 \pi)^{2} B} \sum_{i} S_{i}(\mu)
$$

within the semiclassical approximation. Here, $S_{i}(\mu)$ is the directed area enclosed by the $i$ th segment of the Fermi surface. It is defined as a positive (negative) value when the area enclosed by the segment is filled (empty). The above equation can be cast to the form

$$
\sigma_{x y}^{\mathrm{sc}}(\mu)=-\frac{e^{2}}{h} c_{\mathrm{sc}}(\mu) ; \quad c_{\mathrm{sc}}(\mu)=\frac{\sum_{i} S_{i}(\mu)}{\phi \Omega_{\mathrm{BZ}}},
$$

where $\Omega_{\mathrm{BZ}}=(2 \pi)^{2} / \Omega$ is the area of the Brillouin zone. We found that $c_{\mathrm{sc}}$ completely describes the envelope behavior of 

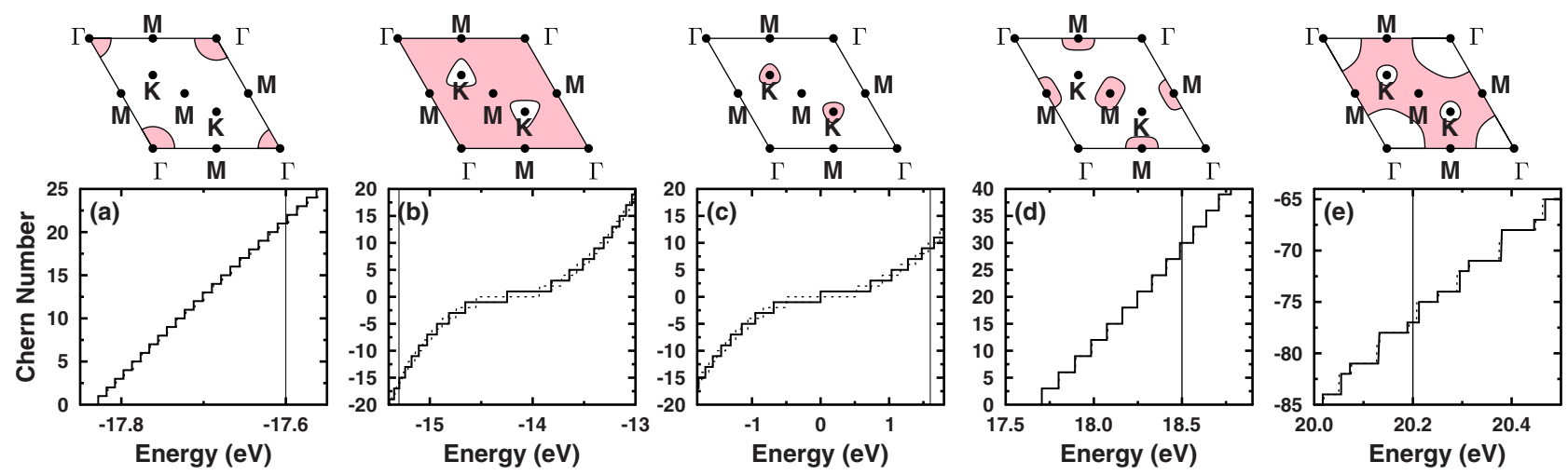

FIG. 3. (Color online) Chern numbers for $\phi=1 / 200$ at several energy regions. The upper panels show the corresponding Fermi surface calculated at the energies indicated by vertical lines. The shaded regions are filled by electrons. The dashed lines indicate the semiclassical estimation $c_{\mathrm{scq}}$ defined by Eq. (8). For (b) and (c), the estimations by Eq. (9) are also plotted but invisible because they trace almost same points with solid lines.

topological number $c_{F}$ for the weak field limit as shown in Fig. 2. While this result may be naively expected as we compare the same physical quantity $\sigma_{x y}$, it is highly nontrivial from the fully quantum-mechanical formulation of Hall conductance. We have succeeded in demonstrating numerically that quantized Hall conductance agrees with the semiclassical limit. ${ }^{21}$ Conversely, this agreement indicates that the formulation based on the Chern number is quantitatively applicable to calculate $\sigma_{x y}$ in the clean limit for realistic energy bands. Thus, this formula may be useful to explore the quantum Hall effect for materials with exotic energy-band structures.

The topological changes in the Fermi surface occur as transitions from electron pockets to hole pockets. At these points, $\sum_{i} S_{i}$ discontinuously decreases with $\Omega_{\mathrm{BZ}}$. Therefore, discontinuity of the Chern number is $\phi^{-1}=q$ and the Hall conductance jumps by $\left(-e^{2} / h\right) \phi^{-1}$ universally.

Another well-known semiclassical formulation of the Hall effect is the Boltzmann theory within relaxation-time approximation. ${ }^{22-24}$ If the relaxation time $\tau$ is approximated by a constant, Hall resistivity $\rho_{x y}^{\mathrm{B}}$ for the weak magnetic field limit does not depend on $\tau$ and is obtained as $^{23}$

$$
\rho_{x y}^{\mathrm{B}}=-\frac{B}{e c} \frac{\eta_{x y z}}{\eta_{x x} \eta_{y y}},
$$

where $\eta_{i i}=\left\langle v_{i}^{2}\right\rangle, \quad \eta_{x y z}=-\left\langle v_{x}\left\{v_{x} \frac{\partial}{\partial k_{y}}-v_{y} \frac{\partial}{\partial k_{x}}\right\} v_{y}\right\rangle, \quad \boldsymbol{v}=\nabla_{k} \boldsymbol{\varepsilon}_{k}$ is a Fermi velocity, and $\langle\cdots\rangle$ implies an average over the Fermi surface. For free-electron dispersion $\varepsilon_{k}=\frac{k^{2}}{2 m}$, the Boltzmann theory gives $\rho_{x y}^{\mathrm{B}}=\frac{1}{n e c} B$, which coincides with the clean limit $\sigma_{x y}^{\mathrm{sc}}=-n e c / B$ as $\rho_{x y}^{\mathrm{B}}=-1 / \sigma_{x y}^{\mathrm{sc}}$. Therefore, it would be interesting to verify whether such correspondence holds for general band structures. The $\rho_{x y}^{\mathrm{B}}$ was evaluated with $\nabla_{k} \varepsilon_{k}$ and $\partial_{i} \partial_{j} \varepsilon_{k}$ computed by a $\boldsymbol{k} \cdot \boldsymbol{p}$ perturbation method (see the Appendix).

In Fig. 2, we compare $\rho_{x y}^{\mathrm{B}}$ with Chern numbers. We found that the Boltzmann theory generally agrees with Chern numbers when the Fermi surface is essentially generated by a single energy band and the chemical potential is not close to van Hove singularities. When multiple bands contribute to the Fermi surface, interband effects become important and two limits of semiclassical theory do not coincide. In such a case, the $\sigma_{x y}$ would sensitively depend on the details of scatterings or relaxations.

Next, we examine the quantized plateaux of $\sigma_{x y}$ in detail. Figure 3 shows an enlarged figure of $c_{F}$ for $\phi=1 / 200$ at several energy regions. At the bottom of the energy bands $\varepsilon \sim-17.8 \mathrm{eV}$, where the Fermi surface consists of an electron pocket around $\Gamma$ points, $c_{F}$ increases with a step of 1 . Therefore, the quantum Hall effect in this region resembles that of free two-dimensional electron gas.

Massless Dirac fermion regimes at $-15.7 \mathrm{eV} \lesssim \varepsilon$ $\lesssim-13 \mathrm{eV}$ and $-3 \mathrm{eV} \lesssim \varepsilon \lesssim 3 \mathrm{eV}$ are shown in Figs. 3(b) and 3(c). In these regions, the Fermi surface is approximated by two massless Dirac cones centered at $K$ points. Here, two Landau bands are grouped together and the energy gap between them are extremely small. Well-developed energy gaps appear between these grouped Landau bands and the Chern number $c_{F}$ increases with 2 between adjacent grouped bands. This behavior may be explained from the existence of two equivalent Fermi-surface segments. However, naive interpretation would give $c_{F}=2 n$ which differs with actual steps $c_{F}$ $=2 n+1$. These plateaux have been theoretically obtained by solving the Dirac equation under magnetic fields ${ }^{10}$ or using the simplified tight-binding model for a carbon $\pi$-orbital. ${ }^{13,14}$ In the present calculation, we included a realistic band structure which deviates from the linear dispersion $\varepsilon_{k} \sim \pm v|k|$. The Chern numbers do not change by such details because the topological number is robust as far as energy gaps do not close. However, the positions of steps are influenced by the band structure. It is most clearly seen in Fig. 3(c) as a violation of the electron-hole symmetry around $\varepsilon=0$. Namely, the widths of plateaux for $\varepsilon<0$ are narrower than those for $\varepsilon>0$. The quantitative explanation of such behavior will be given below.

Near $\varepsilon \sim 18 \mathrm{eV}$, the Fermi surface consists of three electron pockets around $M$ points. We found that three Landau bands are grouped together and the Chern number can be written as $c_{F}=3 n$. More interesting behavior is realized in other energy regions. For example, a Fermi surface around $\varepsilon \sim 20 \mathrm{eV}$ is composed of a hole pocket around $\Gamma$ point and two hole pockets around $K$ point. In this case, $c_{F}$ shows two different steps with increments of 1 and 2 as presented in 
Fig. 3(e). This behavior can be understood by an assumption that $c_{F}$ increases 1 (2) when the semiclassical quantization condition to a Fermi surface around $\Gamma(K)$ points is satisfied.

Let us perform a quantitative comparison with the semiclassical quantization. Onsager's semiclassical quantization condition $^{25}$ can be written as

$$
\frac{S_{i}(\varepsilon)}{\Omega_{\mathrm{BZ}}}=(n+\gamma) \phi .
$$

We assume that Fermi-surface segments individually quantize by the above condition. When the chemical potential is located between quantized levels,

$$
n-1+\gamma<\frac{S_{i}(\mu)}{\Omega_{\mathrm{BZ}}}<n+\gamma,
$$

we further assume that Hall conductance $\sigma_{x y}$ is given by a quantized value $-\frac{e^{2}}{h} n$. Summing up $\sigma_{x y}$ from all Fermisurface segments, we arrive at an expression for the Hall conductance,

$$
\sigma_{x y}^{\mathrm{scq}}(\mu)=-\frac{e^{2}}{h} c_{\mathrm{scq}}(\mu) ; \quad c_{\mathrm{scq}}(\mu)=\sum_{i}\left\lfloor\frac{S_{i}(\mu)}{\phi \Omega_{\mathrm{BZ}}}\right\rfloor+1-\gamma,
$$

where $[\cdots]$ denotes the largest integer not exceeding its argument. If we choose $\gamma=\frac{1}{2}, c_{\mathrm{scq}}$ explains quantized Hall conductance in several energy regions. In Fig. 3 , the $c_{\text {scq }}$ is plotted as dashed lines. They completely agree with $c_{F}$ for Figs. 3(a) and 3(d). When multiple types of Fermi-surface segments exist, the semiclassical quantization deviates from calculated $c_{F}$. It may be caused by multiband effects.

At massless Dirac fermion regions, the $c_{\text {scq }}$ fails to explain $c_{F}$ because semiclassical quantization near massless Dirac cones is different. ${ }^{26}$ The existence of topological phases influences the quantization. ${ }^{27-29}$ Recently, Gosselin et al. ${ }^{30}$ showed that the semiclassical quantization condition of the massive Dirac fermion is described by $\gamma=0$ and 1 . Extrapolating this result to the massless limit, we obtain a modified expression of $c_{\mathrm{scq}}$ for the Dirac fermion:

$$
c_{\mathrm{scq}}^{D}(\mu)=\left\lfloor\frac{S_{D}(\mu)}{\phi \Omega_{\mathrm{BZ}}} \mid+1+\left\lfloor\frac{S_{D}(\mu)}{\phi \Omega_{\mathrm{BZ}}}|=2| \frac{S_{D}(\mu)}{\phi \Omega_{\mathrm{BZ}}} \mid+1,\right.\right.
$$

where $S_{D}(\mu)$ is the area enclosed by a Fermi-surface segment around $K$ points. This equation is also plotted in Figs. 3(b) and 3(c) but almost invisible because it completely traces the Chern number $c_{F}$. Thus, we found an expression of the Hall conductance for the Dirac fermion regime. It completely predicts the positions of quantized plateaux and explains asymmetry around $\varepsilon=0$. If bias voltage toward graphene can be sensitively controlled, the prediction by Eq. (9) may be compared with the experimentally observed plateau structure.

\section{CONCLUSION}

In summary, we demonstrated that the quantized Hall conductance of general band structure can be practically calculated from topological numbers defined to energy bands un- der magnetic fields. Using graphene as an example of multiband models, it was shown that an envelope of quantized Hall conductance coincides with semiclassical expressions when the magnetic field is sufficiently weak. The semiclassical quantization can predict the positions of plateaux for simple bands. For Dirac fermion regions, a modified expression was found to predict the plateaux completely. This expression may be utilized for analysis of experimental data.

\section{ACKNOWLEDGMENTS}

This research by M.A. was partially supported by a Grantin-Aid for Scientific Research from JSPS under Contracts No. 18540331 and No. 17064016. The work by Y.H. was also supported in part by Grants-in-Aid for Scientific Research from JSPS under Contracts No. 20340098 and No. 20654034, and on Priority Areas from MEXT under Contracts No. 220029004 (physics of new quantum phases in super clean materials) and No. 20046002 (novel states of matter induced by frustration).

\section{APPENDIX: $k \cdot p$ PERTURBATION FOR TIGHT-BINDING MODELS WITH OVERLAP INTEGRALS}

The $\rho_{x y}^{\mathrm{B}}$ defined in Eq. (5) was evaluated from $v_{i}=\partial_{i} \varepsilon_{k}$ $\equiv \frac{\partial \varepsilon_{k}}{\partial k_{i}}$ and $\partial_{i} \partial_{j} \varepsilon_{k}$ computed by perturbation expansion. The $\boldsymbol{k} \cdot \boldsymbol{p}$ perturbation for orthogonal tight-binding models ${ }^{31}$ is generalized to the ones with the nonorthogonal basis which we used in this paper. We briefly describe it for the readers' convenience.

Each atom is identified by an index $\eta$ in a unit cell and a translation vector $\boldsymbol{T}$. When the magnetic field is absent, eigenstates $\left|\phi_{n k}\right\rangle$ with wave number $\boldsymbol{k}$ are expanded by Bloch sum of atomic orbitals,

$$
|\boldsymbol{k} \nu\rangle \equiv \frac{1}{\sqrt{N_{c}}} \sum_{\boldsymbol{T}} e^{i \boldsymbol{k} \cdot \boldsymbol{T}}|\boldsymbol{T} \nu\rangle,
$$

as

$$
\left|\phi_{n k}\right\rangle=\sum_{\nu} C_{n k}(\nu)|k \nu\rangle,
$$

where $\nu=(\eta, \xi)$ is a composite index and $n$ is a band index. With the matrix elements of Hamiltonian $\hat{H}$ and overlap integrals written as

$$
\boldsymbol{H}_{\nu, \nu^{\prime}}(\boldsymbol{k})=\left\langle\boldsymbol{k} \nu|\hat{H}| \boldsymbol{k} \nu^{\prime}\right\rangle
$$

and

$$
\boldsymbol{S}_{\nu, \nu^{\prime}}(\boldsymbol{k})=\left\langle\boldsymbol{k} \nu \mid \boldsymbol{k} \nu^{\prime}\right\rangle,
$$

the coefficient vectors $\boldsymbol{C}_{n k}$ of eigenstates $\left|\phi_{n k}\right\rangle$ satisfy

$$
\boldsymbol{H}(\boldsymbol{k}) \boldsymbol{C}_{n \boldsymbol{k}}=\varepsilon_{n}(\boldsymbol{k}) \boldsymbol{S}(\boldsymbol{k}) \boldsymbol{C}_{n \boldsymbol{k}} .
$$

To compute derivatives of $\boldsymbol{\varepsilon}_{n}(\boldsymbol{k})$, we expand $\boldsymbol{H}(\boldsymbol{k}+\boldsymbol{q})$ and $\boldsymbol{S}(\boldsymbol{k}+\boldsymbol{q})$ as

$$
\boldsymbol{H}(\boldsymbol{k}+\boldsymbol{q})=\boldsymbol{H}+\boldsymbol{q} \cdot \nabla \boldsymbol{H}+\frac{1}{2} \sum_{i j} q_{i} q_{j} \partial_{i} \partial_{j} \boldsymbol{H}+\cdots,
$$




$$
\boldsymbol{S}(\boldsymbol{k}+\boldsymbol{q})=\boldsymbol{S}+\boldsymbol{q} \cdot \nabla \boldsymbol{S}+\frac{1}{2} \sum_{i j} q_{i} q_{j} \partial_{i} \partial_{j} \boldsymbol{S}+\cdots .
$$

The actual expressions of these derivatives are easily obtained analytically for tight-binding models. After straightforward perturbation calculations, we obtain

$$
\partial_{i} \varepsilon_{n}(\boldsymbol{k})=A_{i}(n, n)
$$

and

$$
\partial_{i} \partial_{j} \varepsilon_{n}(\boldsymbol{k})=\sum_{m \neq n} \frac{A_{i}(m, n) A_{j}(n, m)+(i \leftrightarrow j)}{\varepsilon_{n}-\varepsilon_{m}}
$$

$$
-\left\{A_{i}(n, n) B_{j}(n, n)+(i \leftrightarrow j)\right\},
$$

$$
+A_{i j}(n, n)
$$

where

$$
\begin{gathered}
A_{i}(m, n) \equiv \boldsymbol{C}_{m}^{\dagger}\left(\partial_{i} \boldsymbol{H}-\varepsilon_{n} \partial_{i} \boldsymbol{S}\right) \boldsymbol{C}_{n}, \\
A_{i j}(m, n) \equiv \boldsymbol{C}_{m}^{\dagger}\left(\partial_{i} \partial_{j} \boldsymbol{H}-\varepsilon_{n} \partial_{i} \partial_{j} \boldsymbol{S}\right) \boldsymbol{C}_{n}, \\
B_{i}(m, n) \equiv \boldsymbol{C}_{m}^{\dagger}\left(\partial_{i} \boldsymbol{S}\right) \boldsymbol{C}_{n} .
\end{gathered}
$$

*arai.masao@nims.go.jp

†hatsugai@sakura.cc.tsukuba.ac.jp

${ }^{1}$ D. J. Thouless, M. Kohmoto, M. P. Nightingale, and M. den Nijs, Phys. Rev. Lett. 49, 405 (1982).

${ }^{2}$ P. Streda, J. Phys. C 15, L717 (1982).

${ }^{3}$ M. Kohmoto, Ann. Phys. 160, 343 (1985).

${ }^{4}$ Q. Niu, D. J. Thouless, and Y.-S. Wu, Phys. Rev. B 31, 3372 (1985).

${ }^{5}$ H. Aoki and T. Ando, Phys. Rev. Lett. 57, 3093 (1986).

${ }^{6}$ B. I. Halperin, Phys. Rev. B 25, 2185 (1982).

${ }^{7}$ Y. Hatsugai, Phys. Rev. Lett. 71, 3697 (1993); Phys. Rev. B 48, 11851 (1993).

${ }^{8}$ T. Fukui, Y. Hatsugai, and H. Suzuki, J. Phys. Soc. Jpn. 74, 1674 (2005) this can be understood as a two-dimensional analog of the King-Smith-Vanderbilt (KSV) formula in Ref. 32.

${ }^{9}$ Y. Zheng and T. Ando, Phys. Rev. B 65, 245420 (2002).

${ }^{10}$ V. P. Gusynin and S. G. Sharapov, Phys. Rev. Lett. 95, 146801 (2005).

${ }^{11}$ K. S. Novoselov, E. McCann, S. V. Morozov, V. I. Fal'ko, M. I. Katsnelson, V. Zeitler, D. Jiang, F. Schedin, and A. K. Geim, Nature (London) 438, 197 (2005).

${ }^{12}$ Z. Zhang, Y.-W. Tan, H. L. Stormer, and P. Kim, Nature (London) 438, 201 (2005).

${ }^{13}$ D. N. Sheng, L. Sheng, and Z. Y. Weng, Phys. Rev. B 73, 233406 (2006).

${ }^{14}$ Y. Hatsugai, T. Fukui, and H. Aoki, Phys. Rev. B 74, 205414 (2006).

${ }^{15}$ R. Saito, M. Fujita, G. Dresselhaus, and M. S. Dresselhaus, Phys. Rev. B 46, 1804 (1992).

${ }^{16}$ H. Min, J. E. Hill, N. A. Sinitsyn, B. R. Sahu, L. Kleinman, and
A. H. MacDonald, Phys. Rev. B 74, 165310 (2006).

${ }^{17}$ H. Song, I. Maruyama, and Y. Hatsugai, Phys. Rev. B 76, 132202 (2007).

${ }^{18}$ M. Taillefumier, V. K. Dugaev, B. Canals, C. Lacroix, and P. Bruno, Phys. Rev. B 78, 155330 (2008).

${ }^{19}$ I. M. Lifshitz, M. Y. Azbel, and M. I. Kaganov, Zh. Eksp. Teor. Fiz. 31, 63 (1956).

${ }^{20}$ I. M. Lifshitz, M. Y. Azbel, and M. I. Kaganov, Sov. Phys. JETP 4, 41 (1957).

${ }^{21}$ In general, the semiclassical theory is justified for small magnetic field where magnetic breakdown does not occur. While the magnetic field used in the calculations exceeds experimentally available field strength, the semiclassical approximation still holds.

${ }^{22}$ T. P. Beaulac, F. J. Pinski, and P. B. Allen, Phys. Rev. B 23, 3617 (1981).

${ }^{23}$ P. B. Allen, W. E. Pickett, and H. Krakauer, Phys. Rev. B 36, 3926 (1987).

${ }^{24}$ N. P. Ong, Phys. Rev. B 43, 193 (1991).

${ }^{25}$ L. Onsager, Philos. Mag. 43, 1006 (1952).

${ }^{26}$ J. W. McClure, Phys. Rev. 104, 666 (1956).

${ }^{27}$ L. M. Roth, Phys. Rev. 145, 434 (1966).

${ }^{28}$ G. P. Mikitik and Y. V. Sharlai, Phys. Rev. Lett. 82, 2147 (1999).

${ }^{29}$ G. Sundaram and Q. Niu, Phys. Rev. B 59, 14915 (1999).

${ }^{30}$ P. Gosselin, H. Boumrar, and H. Mohrbach, arXiv:0805.2455 (unpublished).

${ }^{31}$ M. Graf and P. Vogl, Phys. Rev. B 51, 4940 (1995).

${ }^{32}$ R. D. King-Smith and D. Vanderbilt, Phys. Rev. B 47, 1651 (1993). 